\title{
PricePoints: A System for Aiding Consumers with Grocery Market Shopping
}

\author{
Hmood Al-Dossari, Maha Al-Yahya, Khaled Al-Quraini, Yahya Al-Qahtani, Sultan Al-Barrag \\ College of Computer and Information Sciences \\ King Saud University, Riyadh, Saudi Arabia
}

\begin{abstract}
In grocery markets, price variations are often observed for the same item offered by several groceries. This has led to many consumers paying high prices because of the search costs associated with obtaining price information. In this paper, we present PricePoints, a system to help consumers determine the best available offer and thus save their money. To do so, PricePoints compares several items in a number of grocery stores using multiple criteria in order to guide the consumers to select the best product based on their preferences. The system uses a novel algorithm that is based on multi-criteria including item price, its availability, grocery distance, and reputation. The paper presents the design, implementation, and evaluation of the PricePoints system. The obtained results indicate that the proposed system provides justified and reasonable recommendations for consumers in selecting their preferred groceries.
\end{abstract}

Keywords: E-Commerce; Recommender System, Decision Making.

\section{INTRODUCTION}

People need to buy goods and services constantly, and the search costs to find the best offer are high. Moreover, consumers vary in the way they interpret what constitutes a "best offer" for them. For some, it might be the lowest price; for others, it might be the availability of an item, store location, or store reputation. In a typical grocery item purchase scenario, the consumer usually makes decisions on two levels. The first level consists of determining the preferred item from among many alternatives, and the second level involves finding the best place to purchase it. Usually, this decision is based on price as well as other factors. The process of finding the best offer is highly costly, and services that help save time, effort, and money for the consumer are highly desirable. Moreover, the large number of grocery products available and the different brands have made the decision-making process more complex.

In this paper, we present PricePoints, a system that supports consumers who must decide which grocery items to purchase depending on their preferences (i.e., item price and availability as well as market location and reputation). The system uses a novel algorithm to compare the grocery items, taking into consideration a number of factors (i.e. criteria) that are preferred by that specific consumer.

The rest of the paper is organized as follows: Section 2 presents relevant work. Section 3 presents the research methodology. Sections 4 , and 5 present the system design and components. Sections 6, 7, and 8 present system implementation, evaluation, and discussion, respectively. Section 9 presents the conclusions and directions for further work.

\section{RELATED WORK}

A considerable amount of literature has been published in the area of consumer assistance in e-commerce. One example is a Comparison Shopping Agent (CSA) or shopbot. CSAs are described as systems that "collect product and service information, especially price-related information, from multiple online vendors, aggregate them, and then provide value-added service to online shoppers to assist their online shopping" [1]. One of the earliest shopbots was BargainFinder [2] that enables users to compare music CD prices from several online stores. It mainly operated by comparing prices from several vendors without considering other product information or attributes. ShopBot [3] is an agent that can query vendor websites and extract relevant product information. Unlike BargainFinder, ShopBot initiates the query from the user's browser and not the agent's server. Early CSAs used search engines and screen scrapers to extract relevant product information. In addition, vendor sites were entered by humans as static links.

Comparison shopping sites are usually portals that rely on vendors to provide product information. There might be special relations or partnerships between the CSA and vendors to provide product information. Therefore, although they provide price comparisons, they do not necessarily provide unbiased recommendations (i.e., free of advertisements and commissions). Although the product information is stored in a structured machine-processable form in the vendor databases, shopbots can only access this information by retrieving and parsing web pages, using various methods to extract it. Semantic Web shopbots address the shortcomings of traditional shopbots that are based on screen-scraping. Traditional shopbots extract product information by parsing pages retrieved using screen scrapers [4]. Parsing limits the type, quality, and accuracy of the information being retrieved. 
Semantic Web technologies can extract the semantics of web pages so that extracted product information is more accurate and meaningful. A proposed approach for Semantic Web shopbots was presented in [4]. The approach is based on Web services, Web service registries, and ontologies. The approach was envisioned to address current shopbot problems, mainly the "inability to provide comparisons on attributes other than price and unbiased services."

CSAs have usually focused on comparing individual item prices to find the best price for consumers. However, the comparison agent proposed in [5] considers the total value discounts that could be gained by buying more products from the same retailer. The authors view this problem as an optimization problem of choosing the retailers and items to buy in order to minimize the total buying cost, taking into account the total value discounts offered. They propose a formulation for this problem in a model that could be integrated with CSAs/search engines for multiple item purchases.

In [6], an architecture named ConsiderD was presented that maintains rules in comparison shopping. The rules are extracted from online retailers by humans, automatically expressed in eXtensible Rule Markup Language (XRML), and used to include not just the item price for comparison, but also other information such as delivery cost, delivery options, and shipping rates. The architecture is implemented for the online bookstore domain and was tested on five online bookstores. Results of the evaluation indicate that rule-based computation significantly outperforms the average data-retrieved delivery cost.

Another area relevant to our research is the quality and reputation of services in e-Commerce systems. A reputationbased approach is described in [7] that uses a reputation-based approach to facilitate the protection of consumers. To address the inherent subjectivity of consumer ratings, consumers were asked to provide their expectations alongside their ratings. This approach incorporates expectations into the reputation calculation in order to distinguish between subjective and unfair ratings. The authors observed ratings over time and then divided them into different windows to capture the dynamic behavior of service providers. They then computed the quality of a service for each window. The study presented in [8] evaluates the quality of open web services that are provided by Internet companies in the telecommunications sector to help users choose the best provider depending on the following criteria: execution price, execution time, and reputation. The researchers presented two methods based on those criteria and believe that successful methods depend on the willingness of all users to provide feedback that is correct and accurate about the level of services provided by the suppliers. The researchers recommend gathering more information to better calculate the quality of open web services and predict the best service.

In the area of web services, the study in [9] focuses on methods for computing quality of service (QoS) to enable consumers to make informed choices about the best service. It uses a comparative approach to verify the authenticity, accuracy, and extent of compliance of the published information with the consumers' evaluation of the QoS attributes. The researchers presented methods for service matching, ranking, and selection based on several factors to compute the quality of web services. The approach is also capable of predicting the value of unavailable attributes.

Similarly, the approach presented in [10] computes a personalized QoS ranking based on a prediction framework for cloud services that takes advantage of past consumer experiences. This QoS ranking prediction method does not require additional service invocations to rank. The authors presented two algorithms: cloudRank1 and cloudRank 2 based on employed service, full service, preference function, and confidence value. These algorithms calculate the reference values for these factors and display the arrangement of cloud services to help users to choose the best services.

With regards to consumer behavior when buying online, [11] described factors that affect it. The study compared price and confidence to determine which is the most important for consumers. The researchers studied consumers who were interested in purchasing books by monitoring the opinions of buyers and those who attempted to buy items on book-selling websites. An important finding of this study is that its results might not generalized to other products because different products have different factors that affect it.

While sharing the same aim as the previous studies (i.e., supporting consumer decision-making), our proposed system attempts to address their limitations. It is clear that the success of the majority of the previous proposals mainly depends on the willingness of end-users to give their feedback on the quality of the services that they consume. Additionally, these approaches assume identical items (i.e., all required items are available in all potential markets) when comparing prices, which is unlikely to happen. In doing so, the recommendation and guidance of these approaches could be misleading and inaccurate. In our study, we aim to compare available offers based on our proposed algorithm rather than consumer evaluations. In proposing our solution, we address the problem of having a non-identical list of items, hence providing more useful information to support the consumer decision-making process.

\section{METHODOLOGY}

We elicited and identified requirements by studying the factors that might influence consumers' decisions when purchasing grocery items. An online survey was designed and distributed to over 770 participants (62\% male, 38\% female) to identify the most influential factors that affected their purchase behavior. The survey consisted of eleven multiple choice questions about their behavior when purchasing grocery items.

The results of the survey indicated that more than $70 \%$ of consumers would like an application that helps them purchase grocery items and provides detailed information in terms of price, availability, and market distance.

\section{SYSTEM DESIGN}

The system was designed as an online web-based system. In the following subsections, we describe the design considerations and decisions. 


\subsection{Ranking based on Multi-criteria}

One of the major challenges that we faced in the system design was how to rank the markets based on multiple criteria, specifically, an item's price and availability as well as the market's distance and reputation.

Another important issue is the link between an item's availability and price. That is, we cannot separate one from the other because the price variation of items between different markets depends not only on the total price of the products, but also on the availability of these products in the market. Hence, in order to equitably compare prices, there must be an equivalent availability of the products in all markets. Moreover, a comparison that depends only on the total value of a market is incorrect because there could be a difference between the item's price in these markets (e.g., the price of a packet of rice could be Saudi Riyal (SR) 150 in one market and SR 230 in another) and if we calculate the price of rice with other item prices, we will not notice the large difference between its prices in many markets. To conclude, a traditional comparison of many items based on multiple criteria without considering the relations among them (e.g., the price-availability relation) may produce a misleading result. Hence, a new mechanism is required to provide a reasonable and realistic comparison.

For the purpose of comparing more than one factor at the same time and giving more accurate and realistic outcome, the design and implementation of our system is based on the proposed PricePoints algorithm as described in the next section.

\subsection{PricePoints Algorithm}

In this paper, we propose a PricePoints, an algorithm to compare the prices of items between different markets. The main idea of the PricePoints algorithm is to compare the items of the candidate markets individually rather than collectively. That is, the algorithm starts by taking the first item, compares its price for all markets, and then gives a point to the cheapest market. It then does the same for the next item and continues until it reaches the end of the shopping list.

After comparing all items, the algorithm collects the total points of each market in order to determine the winner (the market with the highest points). The algorithm then removes the market with the highest score from the list and repeats the same process with the remaining markets. Finally, the PricePoints arranges the candidate markets in descending order by score. Figure 1 lists the pseudo-code of the PricePoints algorithm.

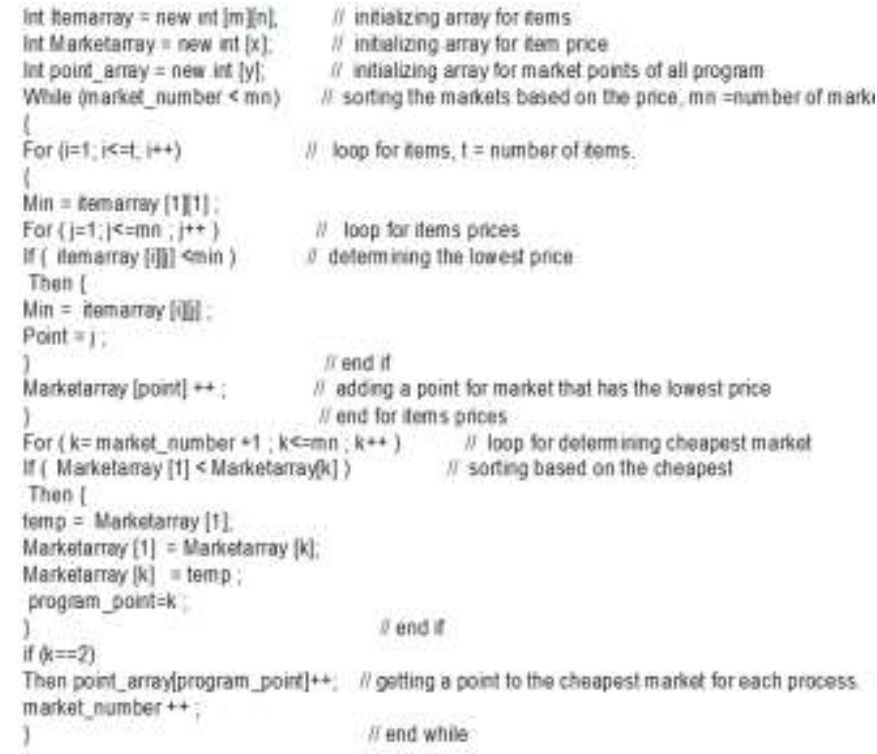

Figure 1. PricePoints Algorithm

By comparing the shopping list item-by-item, we are able to not only consider the relation between availability and price, but also capture any variation in item prices.

\section{SYSTEM COMPONENTS}

Our proposed system comprises three major components: Data Collection, Product Selection and Ranking Calculation. We will describe the three components in details on the following sections.

\subsection{Data Collection}

We assume that there is direct access to market databases. These databases contain the price and number of existing products as well as all relevant information such as category, provider, expiration date, and specification. The Data Collection component extracts product information from several databases and stores them in one central database.

\subsection{Product Selection}

In our system, the consumer can choose a list of grocery products that they wish to purchase and then the system displays all relevant information. Product information includes the four major influential factors: price, availability, distance, and rating.

\section{Price}

Price is the amount money that the consumer must pay the supplier for grocery products such as milk, corn, or rice. The price is the most important factor that influences the consumer when deciding where to buy the required products [12].

\section{Availability}

Availability indicates whether a required product is available in a specific grocery or not. 


\section{Distance}

Distance is computed as the distance from the location of the consumer to the candidate grocery. There are many approaches that can be used to calculate the distance such as Global Positioning System (GPS) or the Google Maps API.

\section{Reputation}

Reputation represents the total points earned by a grocery during the system's usage. At every invocation, the system gives one point to the grocery that gets the highest score when the prices of products in all groceries are compared (i.e., the cheapest grocery). As price is the most important factor in buying decisions, the system computes the grocery reputation based on price. This is a new and unique approach that differs from other reputation approaches that are based on user ratings [13], [14], [15]. These ratings, unfortunately, suffer from subjectivity as well as unfairness. Our proposed approach addresses these limitations by generating the ratings automatically without humans' involvement which may provide fake evaluations [16].

\subsection{Ranking Calculation}

Comparing many items based on multiple criteria is not straightforward. This is because different customers may have different preferences when selecting the best grocery. For example, some may be interested in the cheapest price, while others may be looking for the nearest grocery. The ranking calculation is the final process in our system. After all computations are made, the system displays the best possible offer for the consumer based on their preferences. The following example demonstrates this process.

To demonstrate the process of the PricePoints algorithm, suppose that we have four groceries where each has five products that a consumer SC1 looking for, as shown in Table 1 .

Table 1. Example of groceries with five products.

\begin{tabular}{|l|c|c|c|c|}
\hline & SP1 & SP2 & SP3 & SP4 \\
\hline Milk & 7 & 8 & 9 & - \\
\hline Rice & 1 & 1 & - & 1.5 \\
\hline Tissues & 85 & 70 & 90 & - \\
\hline Detergent & 15 & - & 15 & 14.25 \\
\hline Soda & 5 & 6 & 5 & - \\
\hline Points & $\mathbf{3}$ & $\mathbf{2}$ & $\mathbf{1}$ & $\boldsymbol{1}$ \\
\hline \multicolumn{1}{|c|}{ Total (SR) } & 113 & 85 & 119 & 15.75 \\
\hline
\end{tabular}

It is clear that SP1 is the best grocery in terms of price because it has three product prices that are the lowest (milk, rice, and soda). Hence, SP1 is given three points. In addition, SP2, SP3, and SP4 are given two, one, and one points, respectively. Note that instead of using the absolute total points for each grocery, PricePoints calculates the cheapest as a percentage of the total points as follows:

Grocery percentage $=($ Grocery Points $/$ Total Points $) \times 100$

For example, the percentage of SP1 can be calculated as: SP1 percentage $=100 \times(3 / 5)=60 \%$. The system gives SP1 $60 \%$ of the total points and the percentages of other groceries are calculated with respect to the remaining percentage (40\%). Table 2 shows the other groceries after SP1 was removed from the list.

Table 2. Remaining candidate groceries.

\begin{tabular}{|l|c|c|c|}
\hline & SP2 & SP3 & SP4 \\
\hline Milk & 8 & 9 & - \\
\hline Rice & 1 & - & 1.5 \\
\hline Tissues & 70 & 90 & - \\
\hline Detergent & - & 15 & 14.25 \\
\hline Soda & 6 & 5 & - \\
\hline Points & $\mathbf{3}$ & $\boldsymbol{1}$ & $\boldsymbol{1}$ \\
\hline \multicolumn{1}{|c|}{ Total (SR) } & 84 & 119 & 15.75 \\
\hline
\end{tabular}

Again, from Table 2, it is obvious that SP2 is the second best grocery, as it gets the highest number of points after comparing the prices of all three groceries. To calculate the percentage of SP2, we modified equation (1) as follows:

Grocery percentage $=($ Grocery Points $/$ Total Points $) \times 100 \times$ remaining percentage

Using equation (2), the percentage of SP2 is: $(3 / 5) \times 100 \times$ $0.40=24 \%$. The PricePoints repeats the same process to determine the percentage of SP3 and SP4, and the results are shown in Figure 2.

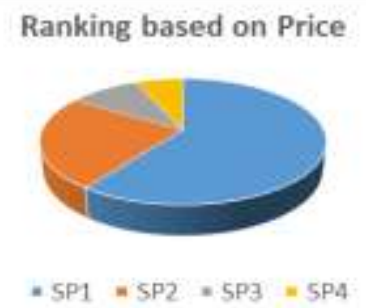

Figure 2. Ranking of the four groceries based on price

After calculating the percentage of each candidate grocery, the PricePoints algorithm arranges the four candidate groceries in descending order and returns the best (cheapest) one to the customer.

\section{Availability}

Ranking the candidate groceries based on availability is a straightforward process. The availability of items for each grocery equals the number of available items divided by the total number of items.

Availability = Available Items/Total Items

For example, the availability of items in SP4 is computed by dividing two (the number of available items: rice and detergent) by five (number of all items) to equal $40 \%$. Table 3 shows the calculation of availability for all groceries. It is obvious that if $\mathrm{SC} 1$ concerns on having all items on a grocery, then the system will nominate SP1 as the best candidate. 
Table 3. Availability of the candidate groceries.

\begin{tabular}{|c|c|c|c|c|}
\hline & SP1 & SP2 & SP3 & SP4 \\
\hline Availability & $100 \%$ & $80 \%$ & $80 \%$ & $40 \%$ \\
\hline
\end{tabular}

\section{Distance}

There are many applications that can calculate the distance such as GPS and the Google Maps API. Table 4 shows an example for the distance between the current customer and the four groceries. If the most important factor for SC1 is how far she is from the candidate groceries, the proposed system will nominate SP3 as the best choice.

Table 4. Distance to the candidate groceries.

\begin{tabular}{|c|c|c|c|c|}
\hline & SP1 & SP2 & SP3 & SP4 \\
\hline Distance (KM) & 1.7 & 2.4 & 0.3 & 4.0 \\
\hline
\end{tabular}

\section{Reputation}

Each time a grocery wins, the system increases its rating factor by 1 . On the other words, the proposed system gives a point to each market that obtains the highest percentage of savings. For example, suppose that we have historical ratings for four markets, as listed in Table 5. It is clear that after 73 transactions, SP1 won 40 times, SP2 won 18 times, SP3 won only once, and SP4 won 14 times.

Table 5. Reputation of the candidate groceries.

\begin{tabular}{|c||c||c||c||c||}
\hline \hline & SP1 & SP2 & SP3 & SP4 \\
\hline \hline Rating & 40 & 18 & 1 & 14 \\
\hline
\end{tabular}

In our running example, after consumer $\mathrm{SC} 1$ finishes their comparison, the system adds one point to the SP1 grocery, if it is the winner, and stores this information in the database. Hence, the rating of SP1 will become 41.

\section{IMPLEMENTATION}

The system was implemented as a web-based system and a detailed test was carried out to ensure that the system functionality is aligned with the proposed design and requirements. The following subsections discuss the ranking of markets based on user preferences: price, availability, distance, and rating.

\subsection{Price}

Assume that the prices of a sample grocery list in four markets are as shown in Table 6.
Table 1. Consumer Requirements

\begin{tabular}{|l|c|c|c|c|}
\hline & SP1 & SP2 & SP3 & SP4 \\
\hline Milk & - & 4 & 7 & 1 \\
\hline Carrots & - & 7 & 15 & 20 \\
\hline Onion & 9 & 9 & 9 & - \\
\hline Video & - & 60 & 140 & 280 \\
\hline Yogurt & 6 & 7 & 8 & 12 \\
\hline Total (SR) & $\mathbf{1 5}$ & $\mathbf{8 7}$ & $\mathbf{1 7 9}$ & $\mathbf{3 1 3}$ \\
\hline
\end{tabular}

We note that the total prices of the items are dissimilar. SP1 has the lowest cumulative total price (SR 15), however, there are three unavailable items (milk, carrots, and a video). This means that SP1 is not necessarily the best choice. For SP2, the cumulative total price (SR 87) is higher than SP1 but it has all the required items. SP4 is the market with the highest cumulative total, furthermore, one of the items (onion) is not available. In contrast SP2 and SP3 have lower cumulative totals and all the required items are available. The results shown in Figure 3 were based on the data in Table 6.

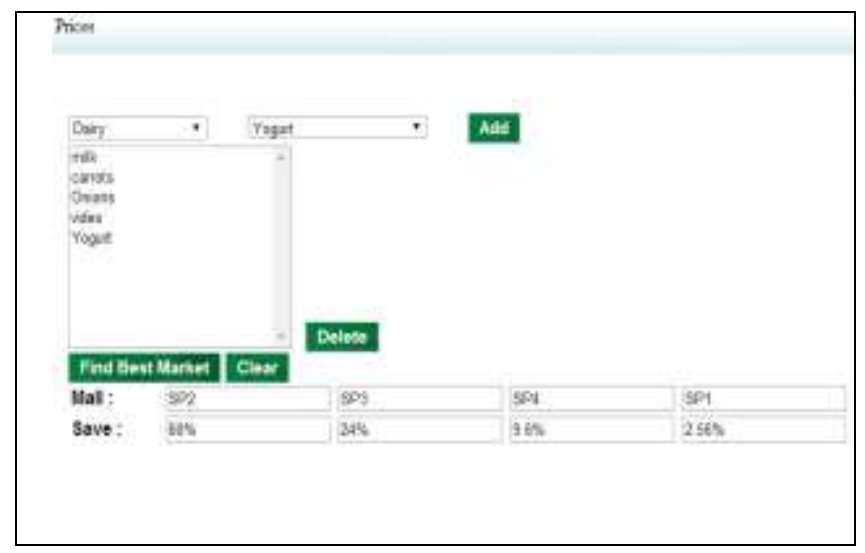

Figure 3. Groceries' Ranking based on Price

The results of the system show that SP2 has the highest percentage of savings $(60 \%)$. Next highest are SP3 (24\%), SP4 (9.6\%), and SP1 (2.56\%). These results are more realistic and can better guide the consumer to the most preferred grocery. In our walking example SP2 is the best choice with regards to the price factor.

\subsection{Availability}

Based on our survey, more than $55 \%$ of participants prefer item availability in the market where they are purchasing grocery items. Our implementation computes availability as the percentage of items that exist in a specific grocery with respect to the total items selected by the consumer. The results from our system are shown in Figure 4. 


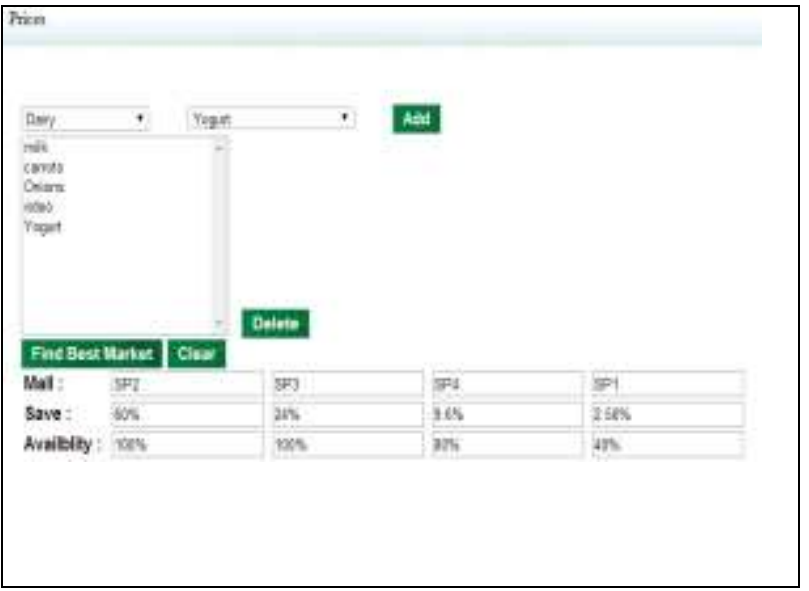

Figure 4. Groceries' Ranking based on Availability

As can be seen in this figure, SP2 and SP3 have 100\% availability because they stock all the grocery items, while SP4 gets $80 \%$ because it does not have any onions and SP4 gets only $40 \%$ because it does not have milk, carrots, or a video.

\subsection{Distance}

The results of our survey showed that $19 \%$ of the participations are concerned by market distance. As we explained in Section 5.2 the system only shows the distance of each grocery. Hence, the consumer determines the nearest grocery. The results of the distance computation in our implementation are shown in Figure 5. As can be seen, SP4 is the nearest at $9 \mathrm{~km}$, followed by SP2 $(12 \mathrm{~km}), \mathrm{SP} 1(15 \mathrm{~km})$, and SP3 $(16 \mathrm{~km})$.

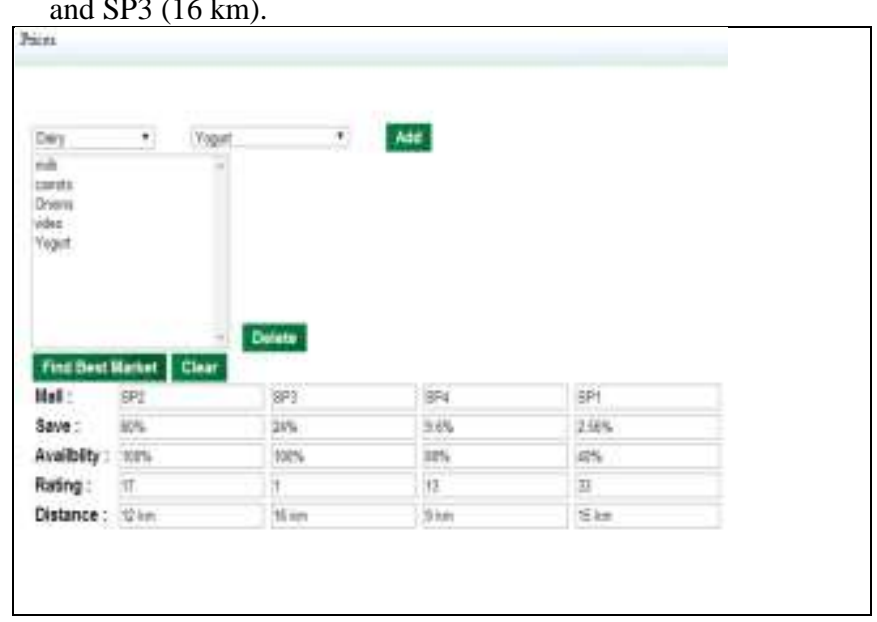

Figure 5. Groceries' Ranking based on Distance

\subsection{Rating}

As explained in Section 5.2, the system gives a point to each grocery that obtains the highest percentage of savings. The results of our survey showed that more than $47 \%$ of participants do not trust the opinions of others, therefore our approach computes these ratings automatically without human intervention.
To illustrate how the system produces the ratings automatically, assume that the current situation for four groceries is shown in Figure 6. It is obvious that SP1 is the best grocery, as it has 35 points. Assume also that we have two consumers SC1 and SC2 each has a different shopping list. The system guides them to the best grocery based on their requirements and consequently adds one point to the best grocery's rating. We set a fixed availability of $100 \%$ for all markets and intentionally assign SP1 the cheapest prices for most of the items.

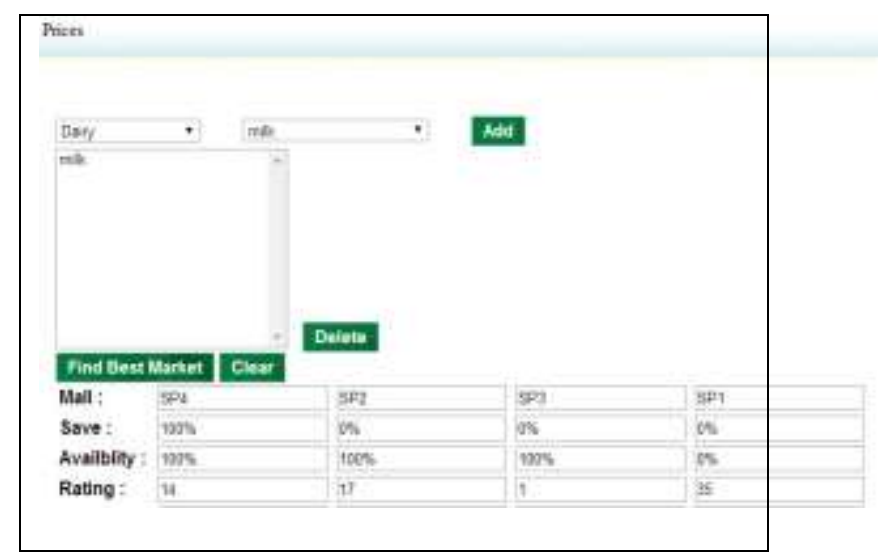

Figure 6. The current rating for the four groceries

Now assume that SC1 has the following shopping list: yogurt, cheese, and soup. When the consumer submits his/her request to the system, the ratings are shown as in Figure 7. It is clear that SP1 obtains $100 \%$ for the savings, indicating that it is the cheapest price for all selected items. Consequently, the system presents it to $\mathrm{SC} 1$ as the best choice and increases its rating by one point to 36, as shown in Figure 7.

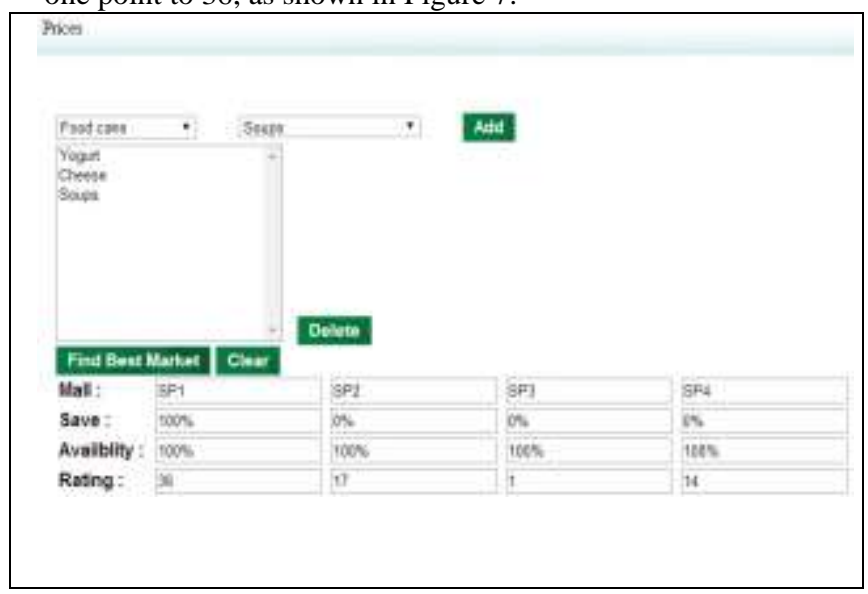

Figure 7. Groceries' rating update after SC1's transaction

The third consumer SC2 submits a request that contains: beans, canned fruit, a TV, and a microwave. The system compares the prices and chooses SP1 as the best grocery in terms of price, as shown in Figure 8. Again, the saving rate for SP1 is the highest (75\%) among the four markets, so it is the winner. The system again increments SP1's rating by one point to 37. 


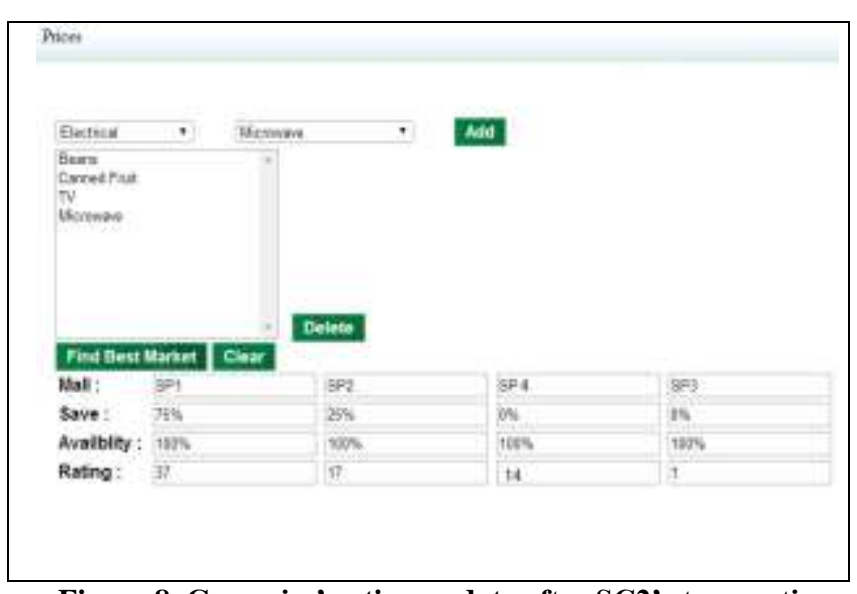

Figure 8. Groceries' rating update after SC2's transaction

\section{Evaluation and Results}

After system development and testing, we evaluated the system to obtain feedback from the consumers on its effectiveness in helping them find the best offer available in a grocery. We designed a survey with 14 statements to elicit participants' views and feedback with respect to the system. A total of 20 users participated in the survey. The statements are shown in Table 7, and the results are shown in the chart in Figure 9.

Table 2. Perception Statements in the survey

\begin{tabular}{|c|c|}
\hline Item & Statement \\
\hline 1 & The system idea is important for the grocery consumer. \\
\hline 2 & The system is easy to use. \\
\hline 3 & $\begin{array}{l}\text { The system covered the most important factors for grocery } \\
\text { consumers. }\end{array}$ \\
\hline 4 & The system raises the level of awareness among consumers. \\
\hline 5 & $\begin{array}{l}\text { The system uses traditional methods in the search for the } \\
\text { cheapest market. }\end{array}$ \\
\hline 6 & The system contributes to saving money. \\
\hline 7 & $\begin{array}{l}\text { This system search is better than traditional methods for } \\
\text { finding the availability of food. }\end{array}$ \\
\hline 8 & The system contributes to saving time and effort. \\
\hline 9 & The system helps identify the nearest market. \\
\hline 10 & $\begin{array}{l}\text { The ratings method used in the system provides a more } \\
\text { credible approach than ratings based on consumers' opinions } \\
\text { and reviews. }\end{array}$ \\
\hline 11 & Rating affects the decision to choose a market. \\
\hline 12 & $\begin{array}{l}\text { The system helps the consumer get a fair deal from the } \\
\text { market owner. }\end{array}$ \\
\hline
\end{tabular}

\begin{tabular}{|l|l|}
\hline 13 & $\begin{array}{l}\text { The system addresses the deficiencies and poor performance } \\
\text { of regulatory organizations for consumer protection. }\end{array}$ \\
\hline 14 & I would recommend the system to friends. \\
\hline
\end{tabular}

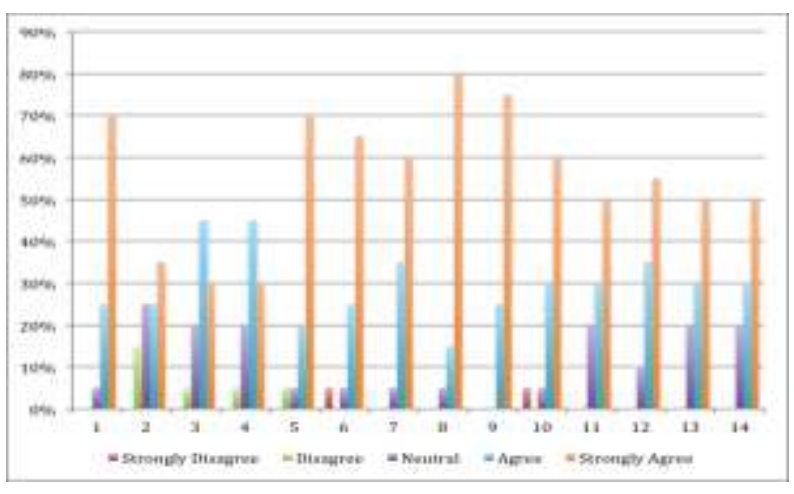

Figure 9. Respondents' perceptions on PricePoints

\section{DISCUSSION}

The results obtained from our evaluation indicate that the system as designed and implemented provides justified and reasonable recommendations for consumers when purchasing grocery items. The results of the survey show that the majority of respondents agreed that there is a need for such a system to help them with their purchases. When asked whether the system was able to consider all relevant factors for consumers when making purchases, the replies varied, but the majority agreed that major factors were considered. When asked about the major issues that concern consumers, that is money savings, time, and effort, and whether the system was able to help them find the best offer, the majority of the respondents agreed.

With regards to the issue of the cheapest market and the availability of items, the majority of respondents agreed that the system excelled over traditional methods. With regards to guidance to the closest available market, survey results show that the majorities indicate that system was able to provide this service. When asked to compare the rating model provided in the system with other traditional rating models based on consumer opinions and reviews, the majority of respondents indicated that this model is better suited to the problem at hand, is more credible, and does have an effect on the decision to choose a specific market.

With regards to the interface design and ease of system use, answers varied. In general, the system was not very complicated, but improvements were needed to the interface to simplify its use and access for non-technical users. When asked if the system provides a solution to address the deficiencies of the regulatory organizations for consumer protection, the majority of respondents agreed. In general, results of the evaluation were positive, as the majority of the respondents $(80 \%)$ indicated that they would recommend such an application to friends. 


\section{CONCLUSION AND FUTURE WORK}

In this paper, we presented a system that helps consumers select the best grocery market based on their preferences (i.e. price, availability, and distance).

The system design is based on incorporating several relevant factors for consumers when making decisions, and combines them in a novel algorithm that we call PricePoints. The system was designed, implemented, and tested with real consumers. An evaluation was carried out to obtain consumer views on the system. Results of the evaluation indicate that the system was useful and provided relevant information that helped them make the best choices for grocery items purchases.

During the design and implementation of the system, we were faced with several challenges. First, it is difficult to get the actual price of grocery items from groceries. A new method of real-time data acquisition and extraction should be included in future designs of our system. Second, we were faced with the challenge of multiplicity, as companies can produce the same product with different specifications and prices. Finally, a major challenge we were faced with is that not all groceries have their data available online, and it was not easy to persuade them to supply us with data about their products and prices.

Results of our evaluation as well as the challenges we faced provide directions we can explore for future. In the current system implementation, we dealt with a small number of items and a small number of factors without information about the quantity required by each item. The PricePoints algorithm can be extended to consider quantity. Another vital improvement to obtain real time data would be to employ "crowdsourcing" or "collaborative participation" methods for data collection. In this approach, the data is not obtained from the vendors, instead ordinary people who actually visit the grocery participate using their mobile devices to add product and cost information to the central database and get, in response, immediate comparison information.

Although our implementation is focused on grocery item shopping, the design of the system is generic and can easily be adapted to other domains such as books, clothing, and hardware.

\section{REFERENCES}

[1] Y. Wan, Comparison-Shopping Agents and Online Small Business, Khosrow-Pour, 2006.

[2] B. Krulwich, The BargainFinder agent: Comparison price shopping on the Internet, Bots and Other Internet Beasties, SAMS NET, 1996.

[3] R.B. Doorenbos, O. Etzioni and D. S. Weld, A scalable comparison-shopping agent for the World-Wide Web, Proc. 1st International Conference on Autonomous Agents, 1997, pp.39-48.
[4] M. Fasli, The next generation of shopbots: Semantic interoperability and personalization, Comp.-Shopp. Serv. Agent Des. Idea Publ. Group, 2009, pp.19-36.

[5] S. Kameshwaran and L. Benyoucef, Optimal buying from online retailers offering total value discounts, Proc. 10th International Conference on Electronic Commerce, 2008, p.21.

[6] G.G. Lim, J.Y. Kang, J.K. Lee and D.C. Lee Architecture and performance of the rule based comparison shopping: Delivery cost experience, Proc. 10th International Conference on Electronic Commerce, 2008, pp.32.

[7] H. Al-Dossari, A reputation-based approach for consumer protection in Saudi Arabia, Proc. 4th International Conference on Computational Aspects of Social Networks (CASoN), 2012, pp.321-326.

[8] Y. Liu, A.H. Ngu and L.Z. Zeng, QoS Computation and Policing in Dynamic Web Service Selection, Proc. 13th International World Wide Web Conference, New York, 2004, pp.66-73.

[9] Z. Xu, P. Martin, W. Powley and F. Zulkernine, Reputation-Enhanced QoS-based Web Services Discovery, Proc. IEEE International Conference on Web Services, 2007, pp.249-256.

[10] Z. Zheng, X. Wu, Y. Zhang, M.R. Lyu and J. Wang, QoS Ranking Prediction for Cloud Services, IEEE Trans. Parallel Distrib. Syst., Vol.24, No.6, 2013, pp.1213-1222.

[11] H.-W. Kim, Y. Xu and S. Gupta, Which is more important in Internet shopping, perceived price or trust?, Electron. Commer. Res. Appl., Vol.11, No.3, 2012, pp.241-252.

[12] S.A.G. Katherine Isselmann Disantis, What 'Price' Means When Buying Food: Insights From a Multisite Qualitative Study With Black Americans., Am. J. Public Health, 2013.

[13] T.D. Huynh, N.R. Jennings and N.R. Shadbolt, An integrated trust and reputation model for open multiagent systems, Auton. Agents Multi-Agent Syst., Vol.13, No.2, 2006, pp.119-154.

[14] S. Reece, A. Rogers, S. Roberts and N.R. Jennings, A Multi-Dimensional Trust Model for Heterogeneous Contract Observations, Proc. 22nd AAAI Conference on Artificial Intelligence, 2007, pp.128-135.

[15] G. Zacharia, Trust management through reputation mechanisms, Appl. Artif. Intell., Vol.14, 2000, pp.881907.

[16] A. Jøsang, R. Ismail and C. Boyd, A Survey of Trust and Reputation Systems for Online Service Provision, Decis. Support. Syst., Vol.43, No.2, 2007, pp.618-644. 